\title{
Antioxidant Effects of Epigallocatechin Gallate in Cerulein-Induced Pancreatitis
}

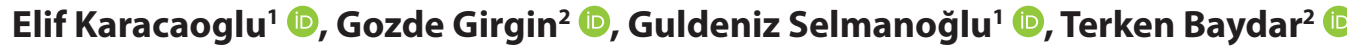

'Hacettepe University, Faculty of Science, Department of Biology, Ankara, Turkey

${ }^{2}$ Hacettepe University, Faculty of Pharmacy, Department of Toxicology, Ankara, Turkey

ORCID IDs of the authors: E.K. 0000-0003-3426-4584; G.G. 0000-0002-7051-0490; G.S 0000-0001-6565-736X; T.B 0000-0002-5497-9600

Please cite this article as: Karacaoglu E., Girgin G., Selmanoglu G., Baydar T. Antioxidant Effects of Epigallocatechin Gallate in Cerulein-Induced Pancreatitis. Eur J Biol 2019; 78(2): 125-132. DOI: 10.26650/EurJBiol.2019.0035

\begin{abstract}
Objective: Acute pancreatitis (AP) is an inflammatory disease of the pancreas resulting from auto-activation of digestive enzymes and damage to the pancreatic parenchyma. Reactive oxygen species (ROS) play an important role in the progression of AP. In the present study, we aimed to evaluate epigallocatechin-3 gallate (EGCG) in reducing the inflammatory reaction and tissue damage in experimental AP rat model.
\end{abstract}

Materials and Methods: Amylase, tumor necrosis factor-alpha (TNF-a) and interleukin-6 (IL-6) levels were measured. Histopathological, immunohistochemical analyses of apoptotic cells, CD-8a and CD-68 were performed. Superoxide dismutase (SOD), catalase (CAT) and glutathione S-transferase (GST) were determined in hemolysates.

Results: Cerulein+EGCG treatment did not cause decreases in the amylase levels. IL-6 levels decreased in cerulein+EGCG group, however, TNF-a levels increased. No changes were observed in SOD activity by EGCG treatment, CAT and GST activities increased. EGCG treatment caused severe edema, inflammation and fat necrosis after cerulein-induced pancreatitis. Apoptosis in pancreas, CD8- $a$ and CD-68 positive cells increased in EGCG treatment after pancreatitis induction.

Conclusion: It may be suggested that EGCG showed a pro-oxidant effect, in contrast to the expected in the pancreatitis model when compared to a positive control. It can be concluded that overconsumption of EGCG should be avoided in pancreatitis conditions.

Keywords: Acute Pancreatitis, Reactive Oxygen Species, Epigallocatechin-3 Gallate, N-acetylcysteine, Antioxidant Effect

\section{INTRODUCTION}

Acute pancreatitis (AP) is an inflammatory disease of the pancreas resulting from the auto-activation of digestive enzymes and damages to the pancreatic parenchyma as the result of auto-digestion. Various causative factors underlie the disease process, including gallstone disease, alchohol abuse, hyperlipidemia, drugs (azathioprinei sulphonamides, estrogens), autoimmune diseases etc. (1). Eighty per cent of the cases are mild/edematous pancreatitis, and resolve completely with proper treatment modalities (2). On the other hand, the remaining one fifth of the cases progress to severe AP (SAP), and have a mortality rate of $20-30 \%$ (1-3). The main pathophysiologic event in the initiation and progression of pancreatitis is the digestive enzyme auto-activation and acinar cell damage leading to inflammation (2). Trypsin is the main pancreatic enzyme that is activated, and is responsible for the parenchymal damage and also for activation of the inflammatory cascade $(2,3)$. It activates the kallikrein and kinin systems, and also activates the coagulation and/or fibrinolysis cascade (4). Inflammation and acinar cell damage is actually an overlapping event. Local inflammation causes an upregulation in the expression of the adhesion molecules in the capillary endothelium, leading to the increased 
migration of the leukocytes to the region (4). The migrating neutrophils will be activated in the pro-inflammatory milieu, and start degranulation (2-6). This leads to the production and release of various mediators, such as phospholipase $A 2$, nitric oxide and oxygen radicals. All those result in an increase in the severity of the inflammation, and it is usually seen with $\operatorname{SAP}(5,6)$. Elucidation of the factors that cause the progression of the potent inflammatory response and furthermore, modulation of this response, have been the subject of current pancreatitis research. In pancreatitis, once the disease process progresses to SAP, various organ systems are affected by this systemic inflammatory process $(2,5,7,8)$.

Reactive oxygen species (ROS) seem to play a crucial role in the pathogenesis of AP (9). The leukocytes isolated from the patients with pancreatitis showed enhanced capabilities for production and release of $\operatorname{ROS}(9,10)$. The enhanced production of ROS, not only leads to severe organ damage, but also causes activation of nuclear factor kappa-light-chain-enhancer of activated B cells $(\mathrm{NF}-\boldsymbol{x} \mathrm{B})$ pathway activation $(10,11)$. Since ROS causes both tissue damage that potentiates the inflammatory process, and enhanced pro-inflammatory cytokine production through certain cellular pathways, blocking the ROS may be a valid treatment strategy for the treatment of AP (10).

Experimental models for AP provide the exact mechanism of the disease and developing new therapeutic strategies. Recently, several animal models have been produced for AP, including non-invasive and invasive methods (12). In the present study, we used the hormone-induced model (cerulein as a cholecystokinin-pancreaozymin analogue), which is one of the non-invasive methods for induction of AP. This experimental method was chosen due to several advantages, such as being an easy simple method to perform. Additionally, the ceruleininduced AP model was reported to be a useful method, due to healing process which begins after the disease induction agent is discontinued (12).

Epigallocatechin-3 gallate (EGCG) is a naturally occurring polyphenol that belongs to the broad family of chemical compounds called the flavonoids $(13,14)$. Flavonoids are abundant in various foods such as apples, grapes, cherries, berries, while EGCG is a polyphenol that is mainly found in cacao and its products, and especially in green tea $(13,14)$. EGCG, like all polyphenols acts through antioxidant and non-antioxidant mechanisms. Antioxidant mechanisms aim to restore the redox balance and counteract the ROS activity in organisms, and repair oxidant-induced injury (15-17). However, non-antioxidant mechanisms include the oestrogen receptor signalling, cell signalling cascade and cell cycle control pathways (18). Green tea polyphenols have high antioxidant capacity, and these phenolic compounds proved to exert pro-oxidant effects and, by means of their pro-oxidant effect, they were suggested as a potential prevention mechanism for cancer (19). Studies from cancer research have shown that EGCG inactivates the signal transduction and transcription (STAT) pathways (20). Furthermore, it also plays a role in the inactivation of anti-tumour immunity.
Persistent STAT3 activation causes a tumour promoting inflammation through the activation of NF- $\varkappa B$, interleukin-6 (IL6) family of cytokines and gp130, Janus Kinase (JAK) pathway (21). Additionally, EGCG binds the $\mathrm{p} 65$ subunit of NF- $\boldsymbol{x} B$ and inhibits the pathway, and causes a reduction in the proinflammatory cytokine production. The above mentioned action mechanism of EGCG emphasizes its capability to reduce inflammation (22). Therefore, it can reduce the main pathophysiologic event, inflammation in AP, and reduce tissue damage and hence, can decrease the morbidity and mortality resulting from this serious illness. On the other part, $\mathrm{N}$-acetylcysteine (NAC), which plays a crucial role in the formation of glutathione as a powerful antioxidant in the body, has been shown to prevent the increase in the cytosolic calcium status, and reduce the accumulation of enzymes in acinar cells during AP (23). Green tea has been demonstrated as a polyphenol-rich extract with a high content of cathechins, including epicathechin, epicathechin-3-gallate, EGCG and epigallocathechin (24), and EGCG was known to have potent antioxidant properties among other cathechins (25). Although effects of green tea extract on cerulein-induced pancreatitis was studied in a mice model previously (11), in that study, the authors evaluated the whole green tea extract rather than a flavonoid. So, it is necessary to study which flavonoids in the green tea extract caused ameliorative effects. So, we intended to evaluate EGCG which is the most abundant flavonoid found in the green tea. The main aim of this study was to evaluate ECGC in reducing the inflammatory reaction and tissue damage in an experimental AP rat model.

\section{MATERIALS AND METHODS}

\section{Animals}

All experiments were performed in accordance with the protocols approved by the veterinarian. This study was performed by the official permission of the Hacettepe University Ethical Committee for the Protection of Animals in Research (\#2008/54). Male Wistar rats (approximately weighing 300-350 g) were housed in polycarbonate cages in a controlled environment with12:12-hours light-dark cycles. The rats were given standard laboratory chow and water ad libitum.

\section{Chemicals}

Cerulein ( $\geq 95 \%$ ) was provided by Sigma-Aldrich (St. Louis, MO, USA). Both of EGCG, and NAC were obtained from Sigma-Aldrich (St. Louis, MO, USA), and each stock solution with a $100 \mathrm{mg} / \mathrm{kg}$ concentration was prepared in water. All other chemicals and reagents were provided by Sigma-Aldrich (St. Louis, MO, USA).

\section{Induction of Acute Pancreatitis}

Rats were injected intramuscularly with ketamine $(50 \mathrm{mg} / \mathrm{kg})$ and xylazine $(5 \mathrm{mg} / \mathrm{kg}$ ) for anesthesia. After anesthesia, an intravenous catheter was placed into right jugular vein. Rats were injected twice into the catheter at a 1-h interval, with a supra-maximally stimulating dose of cerulein to elicit AP. Rats in the control group received similar injections of saline solution. The animals were injected with saline following the induction of AP. 


\section{Experimental Groups}

The treatment was started with EGCG and NAC following the induction of AP in order to simulate the actual clinical scenario. Therefore, 20 animals were randomized into 4 groups including 5 animals in each group.

Pancreatitis was induced by cerulein at a dose of $5 \mu \mathrm{g} / \mathrm{kg} / \mathrm{h}$, established following intravenous infusion from the catheter inserted into the internal jugular vein as standard (13). Rats with cerulein-induced pancreatitis were treated with EGCG or NAC at doses of $100 \mathrm{mg} / \mathrm{kg}$ i.p.at 6 and 18 hours. The dose selection of EGCG was based on the previous study (26). NAC was used as a positive control. Laparotomy was performed in all groups, including the control group at 24 hours. Groups were as follows:

The control group consisted of the sham group; rats were injected with saline in two doses 1 hour a part. The cerulein group was the AP group, induced by Cerulein. The cerulein+NAC group consisted of the NAC treated pancreatitis group and the cerulein+EGCG group consisted of the EGCG treated pancreatitis group. At the end of the experiment, the animals were sacrificed under anaesthesia by opening the thoracic cavity and also drawing blood via the inferior vena cava.

\section{Management and Harvest of the Samples}

Venous blood samples and pancreatic tissues were dissected from the animals. Pancreatic tissues were fixed in 10\% neutral buffered formalin for immunohistochemistry and Bouin's solution for histopathology. Blood samples were taken and placed in heparinized tubes, and centrifuged at $3500 \mathrm{rpm}$ for 15 min. Erythrocytes were washed with PBS, and hemolysates were prepared with the addition of cold deionized water, and samples were centrifuged for the removal of cellular debris. The supernatants were used for the enzyme assays. All supernatant samples were stored at $-20^{\circ} \mathrm{C}$ until further analyses.

\section{Histopathology}

Pancreas tissues of each rat were dissected, then observed grossly and weighed. Relative organ weights (organ weight/ body weight) were calculated. Pancreas tissues were fixed in Bouin's solution, dehydrated in increasing alcohol degrees and embedded in paraffin blocks. $5 \mu \mathrm{m}$-thick sections were obtained, and were tissues stained with H\&E for the light microscopy. Histological evaluation was performed according to Schmidt criteria (Table 1).

\section{Immunohistochemistry of CD8- $\alpha$ and CD68}

Immunohistochemical analyses were principally performed by the streptavidin-biotin amplification method. Monoclonal antibodies were against formalin fixed CD8-a (Santa Cruz Biotechnology, INC, 6A242) and CD68 (Santa Cruz Biotechnology, INC, 6A324) transmembrane proteins. Briefly, portion of the pancreas tissues were fixed in $10 \%$ neutral buffered formalin, dehydrated in increasing alcohol degrees and embedded in paraffin blocks. Then $5 \mu \mathrm{m}$-thick sections were replaced on polyL-lysine coated slides. After sections were deparaffinized, tissue sections were treated with citrate buffer at $95^{\circ} \mathrm{C}$ for $20 \mathrm{~min}$ for antigen retrieval. Sections were washed with PBS with $0.01 \%$ Triton X-100 and treated with $0.3 \%$ hydrogen peroxidase for 15 min at room temperature, then incubated with ultra $V$ block for $10 \mathrm{~min}$. The sections were incubated with mouse CD8-a against rat (1:50 dilution), and with mouse CD68 against rat (1:25 dilution) at $+4^{\circ} \mathrm{C}$ overnight. Peroxidase labelling was carried out using the Ultravision Polyvalent (Rabbit-Mouse) HRP kit (Thermo Sceintific/ Lab Vision), and the positive stainings were visualized by using DAB kit (Thermo Scientific/Lab Vision) to display the reaction product with a brown colour. Then, counterstaining was performed by Haematoxylin staining. Ten random areas were selected at x100 magnification, and CD8- $a$ and CD68 positive cells were counted. CD8- $a$, and CD68 were expressed as positive cell numbers per square millimetre.

\section{TUNEL Assay}

$10 \%$ neutral buffered formalin fixed sections were deparaffinised and dehydrated. Then, sections were digested by Proteinase $\mathrm{K}$ for 15 minutes at room temperature. The endogenous peroxide was quenched in $3 \%$ hydrogen peroxide. Apoptotic nuclei of the pancreas were performed by in situ terminal deoxynucleotidyl transferase (TdT)-mediated deoxyuridine triphosphate (dUTP)biotin nick end-labelling (TUNEL) using Apoptag ${ }^{\oplus}$ Plus kit (Milipore, S7100 EMD Millipore). Counterstain was performed by $0.5 \%$ methyl green. Apoptotic cells were quantified by counting the number of TUNEL positive cells per square millimeter. For each section, 10 areas were randomly selected at $x 100$ magnification per animal and then, the numbers of apoptotic cells were calculated per one square millimeter.

\section{Enzyme Assays}

Catalase (CAT) and superoxide dismutase (SOD) enzyme specific activities were performed as previously described (27). Briefly, blood samples were centrifuged, and separated erythrocytes were washed with PBS. Supernatant samples of hemolysates

Table 1. Schmidt Criteria for histopathological scoring

\begin{tabular}{lllll}
\hline Pathological Grade & $\mathbf{0}$ & $\mathbf{1}$ & $\mathbf{2}$ & $\mathbf{3}$ \\
\hline Edema & None & Interlobular & Intralobular & Inter acinus \\
\hline Acinar cell degeneration & None & Focal $(<5 \%)$ & Sublobular $(5 \%-20)$ & Lobular $(>20 \%)$ \\
\hline Inflammation & None & Mild & Moderate & Severe \\
\hline Hemorrhage & None & Mild & Moderate & Severe \\
\hline
\end{tabular}


were used for the experiments for SOD, CAT and glutathione-Stransferase (GST) antioxidant enzyme activity measurements. Total protein component was measured in order to calculate the specific enzyme activities. Amylase, IL-6 and tumour necrosis factor alpha (TNF-a) in serum samples were measured with commercial ELISA kits.

\section{Statistical Analysis}

All results were expressed as mean \pm standard error. Differences between groups were analysed by non-parametric Kruskal-Wallis variance analyses. Mann-Whitney U-test was performed for independent groups with $\mathrm{p} \leq 0.05$ significance levels.
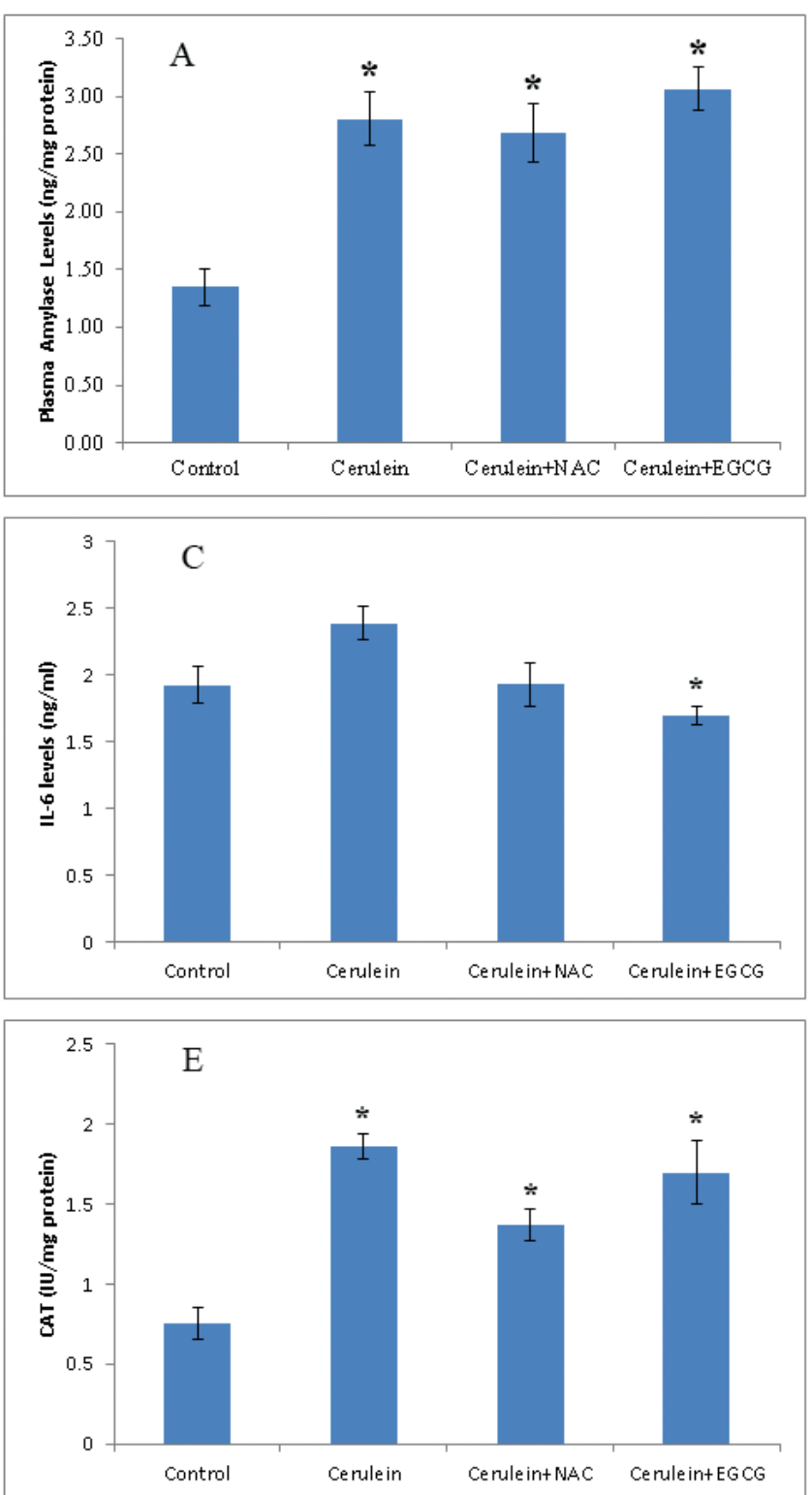

\section{RESULTS}

Plasma serum amylase activities were shown in Figure 1A. According to the amylase activity results, the amylase activity in cerulein, cerulein+NAC and cerulein+EGCG groups was statistically increased when compared to control group $(p<0.05)$.

Serum inflammatory cytokine levels were measured, and the results were shown in Figure 1B-1C. While IL-6 levels statistically decreased, TNF-a levels of cerulein+EGCG group were statistically increased when compared to the control group $(p=0.009, p=$ 0.009). Additionally, TNF-a levels of cerulein+EGCG group were also significantly increased compared to the cerulein and cerulein+NAC groups (respectively, $p=0.04, p=0.02$ ).
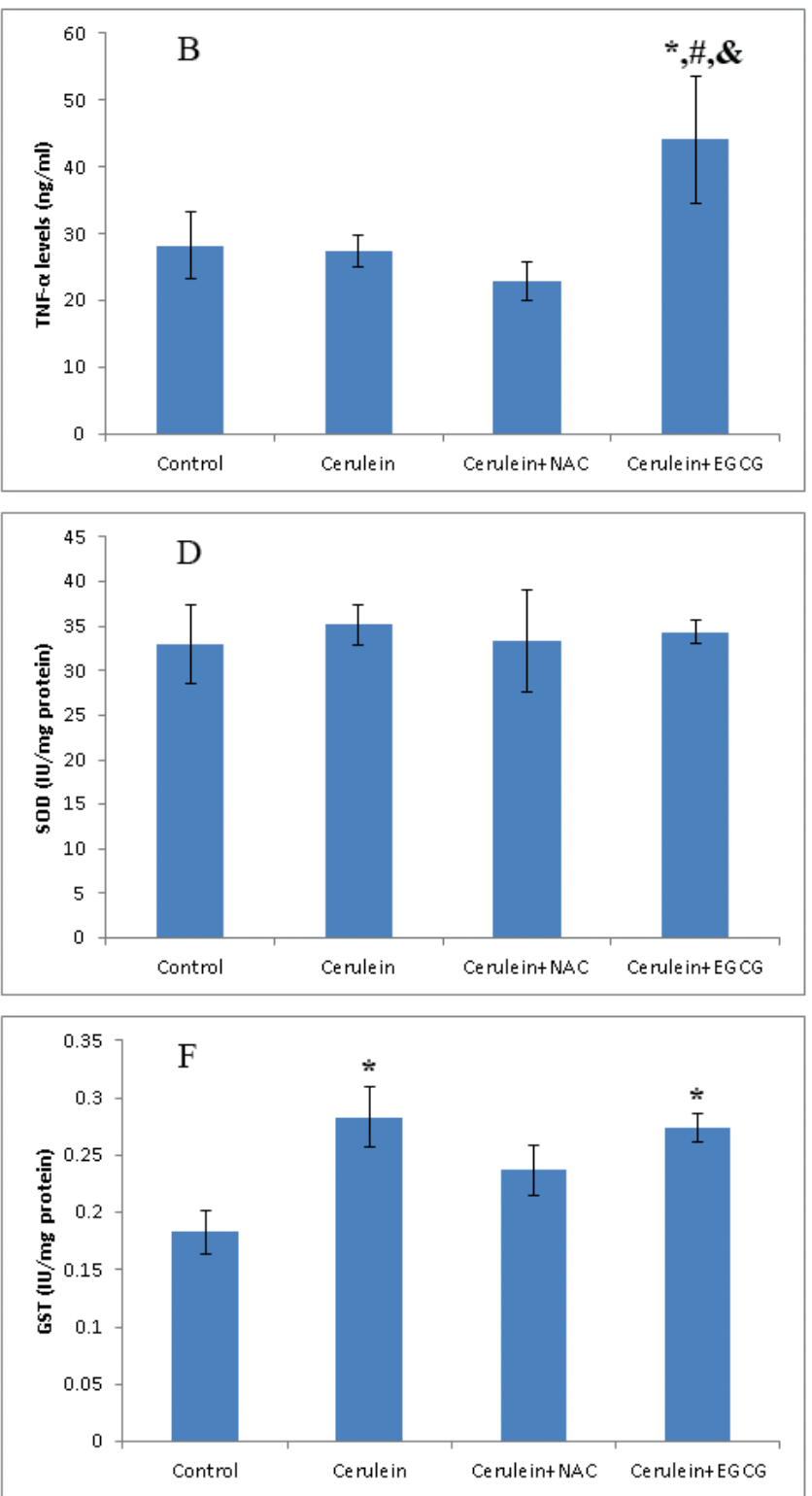

Figure 1. Enzyme activity and inflammatory cytokine results of rats belong to all groups. A) Plasma amylase activity, B) Serum TNF-a levels, C) Serum IL-6 levels, D) SOD activities, E) CAT activities, F) GST activities. *Statistically significant from control group, "Statistically significant from cerulein group, "Statistically significant from cerulein+NAC group ( $\leq \leq 0.05)$. Each bar represents mean \pm standard error. 
Activities of antioxidant enzymes, including SOD, CAT and GST were performed, and results were shown in Figures 1D-F. SOD activities did not differ among groups. CAT activities increased in the cerulein group, as well as in the cerulein+NAC and cerulein+EGCG groups compared to control group. GST activities of the cerulein group, as well as the cerulein+EGCG group were found significantly increased compared to the control group (respectively, $\mathrm{p}=0.03$ and $\mathrm{p}=0.01$ ).

Pancreases of the rats in all groups were evaluated histopathologically to determine the pancreatitis severity according to Schmidt criteria. Pancreas tissues were evaluated for the severity of edema, inflammation and fat necrosis. Histopathological results were shown in Figures 2-3A. The control group had no pathological findings. Edema in cerulein (Figure 2B) and cerulein+EGCG groups was found statistically increased compared to the cerulein+NAC group (Figure 3A). Groups were also compared in terms of inflammation and fat necrosis (Figure $3 \mathrm{~A})$. Both the cerulein and cerulein+EGCG groups were statistically increased compared to the cerulein+NAC group.
According to TUNEL assay results, apoptotic cells increased significantly in the cerulein+EGCG group compared with the cerulein+NAC group (Figure 3). CD8-a positive cells in the cerulein+EGCG group increased statistically compared with the cerulein+NAC $(p=0.035)$. Results were shown in Figure 3B. Although CD68 positive cells increased in the cerulein+EGCG, no statistically significance was found between groups.

\section{DISCUSSION}

In recent decades, studies on oxidative stress, which plays a crucial role in the development of several pathologies, have drawn attention of scientists to understand the relationship between oxidative stress and diseases, and the struggles to develop new treatment strategies. AP is a complex disease that brings about several complications, and associated with a high mortality rate (28). Pathophysiology of AP was mainly attributed to oxidative stress and therefore, trials of several antioxidant agents came into prominence for therapy.
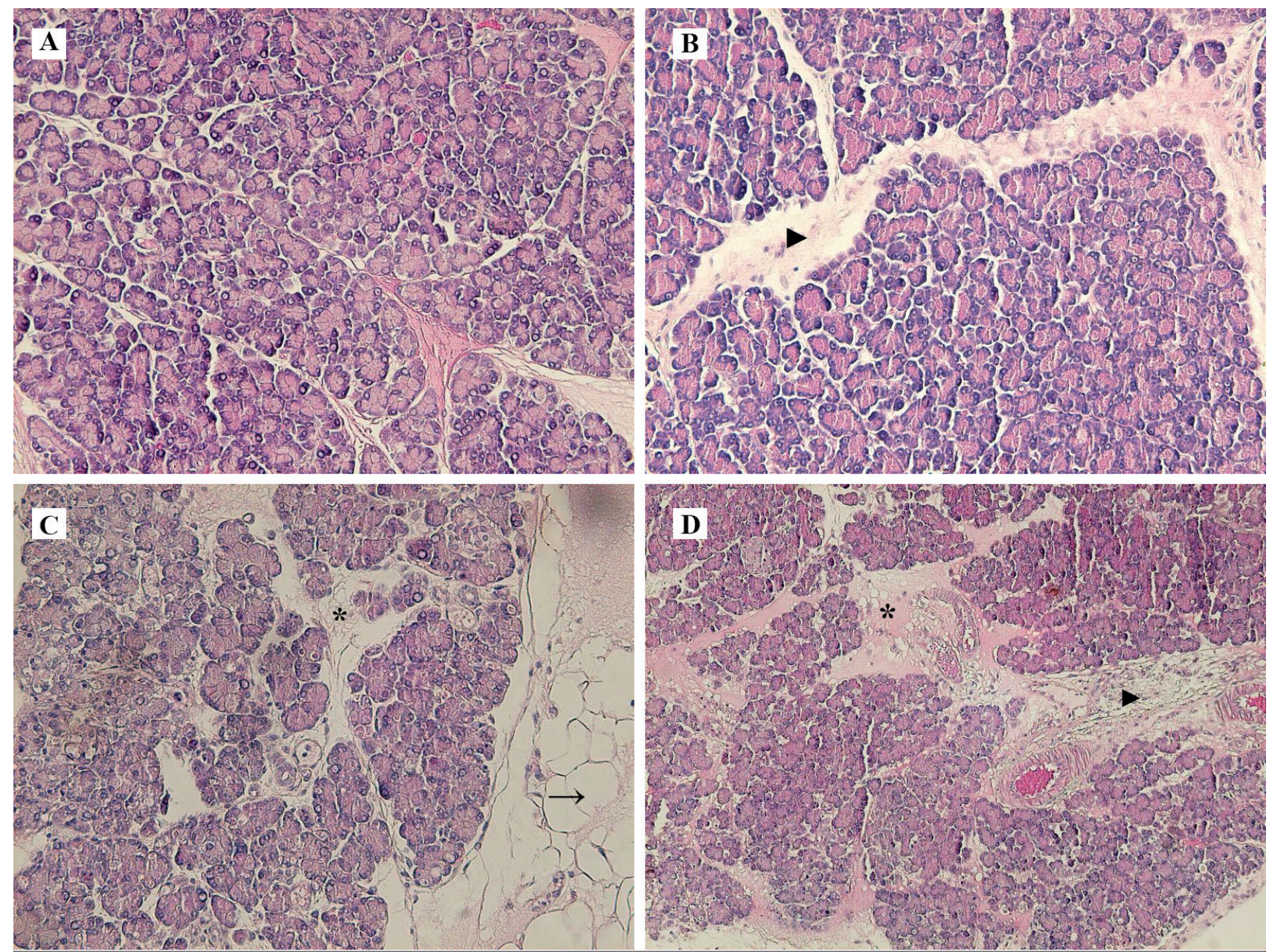

Figure 2. Histopathological findings according to Schmid criteria, H\&E staining. A) Control group, x100; B) Cerulein group, interlobular edema $(\rightarrow)$, x100; C) Cerulein+EGCG, intralobular edema $(*)$, and fat necrosis $(\rightarrow), x 100 ;$ D) Cerulein+EGCG, intralobular edema $(*)$ and inflammation $(>)$, $\times 50$. 

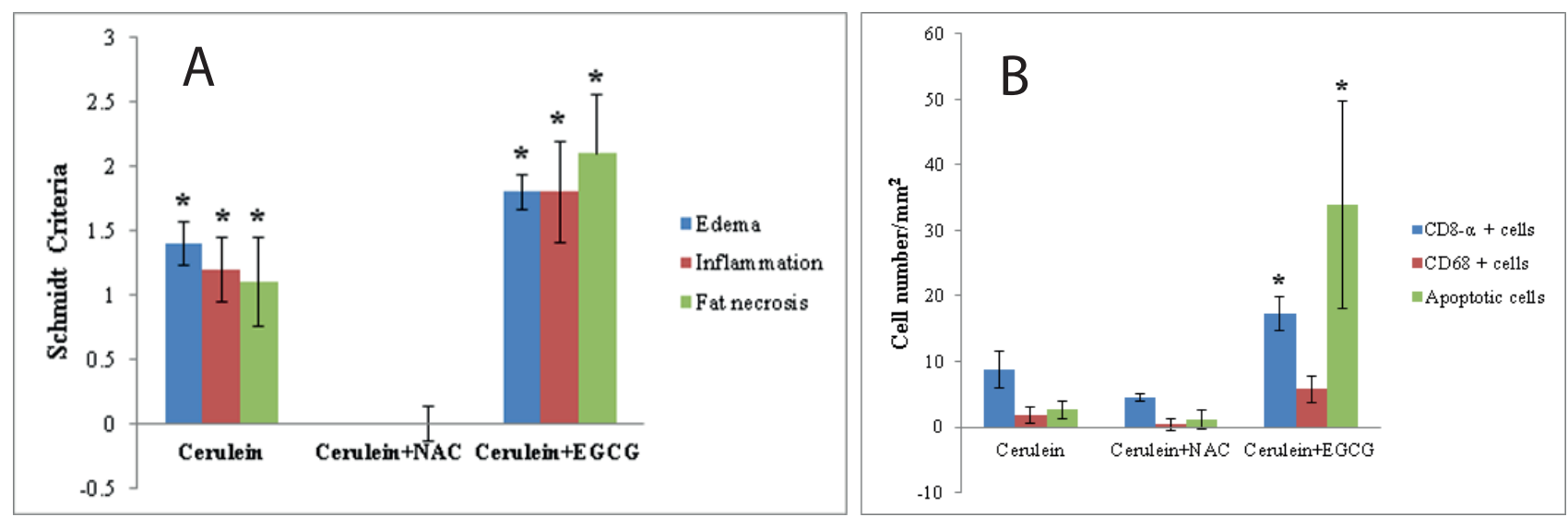

Figure 3. A) Graph of histopathological evaluations according to Schmidt criteria. *Statistically significant from Cerulein+NAC group, ( $\mathrm{p} \leq$ 0.05). Each bar represents mean \pm standard error. B) Graphical analysis of immunohistochemical stainings, \#Statistically significant from Cerulein+NAC group, $(\mathrm{p} \leq 0.05)$. Each bar represents mean \pm standart error.

Cerulein-induced pancreatitis was proven to be one of the alternative experimental pancreatitis models, and best characterized by exerting similar pathophysiological characteristics (29). In normal physiological conditions, digestive enzymes are known to be stored in acinar cells as proenzymes in zymogen granules. When animals were exposed to high dose cerulein which is a cholecystokinin (CCK) analogue, it was shown to cause induction of exocytosis of zymogen granules and consequently, excessive secretion of digestive enzymes. However, after stimulation by excessive CCK when bound to low affinity CCK receptor, digestion enzyme secretion is inhibited (30). In previous studies, it was indicated that induction of pancreatic enzyme activated by cerulein in 30 min after intravenous injection. Hyperamylasaemia, as well as common histopathological changes, including inflammatory cell infiltration, active digestive enzyme in pancreas and edema are the major findings of the ceruleininduced pancreatitis model (30). In the present study, cerulein caused increases in serum amylase levels, as well as histopathological changes, indicating that the AP model was successful. In the present study, while serum amylase level decreased in the cerulein+NAC group, it increased in the cerulein+EGCG treatment groups compared to the cerulein group. Although NAC seemed to be protective effects against pancreatitis, EGCG treatment worsened the pancreatitis scenario.

Oxidative stress is caused by the impairment of the balance between ROS and the antioxidant system of the body (31). Oxidative stress has been reported to play an important role in the pathophysiology of several diseases, resulting in high mortality such as in AP, endotoxic shock as well as sepsis. Besides, the body has a defence mechanism that scavenges the excess free radicals. Increases in SOD and CAT activities are consistent with ROS, and it was shown that SOD and CAT activities elevated in SAP (32). GST is an antioxidant enzyme and plays important role in oxidative stress situations (33). In the present study, SOD activities did not change between groups. However, CAT and GSH enzyme activities in the cerulein+NAC and cerulein+EGCG groups were found higher than in the control group. It should be stated that NAC treatment may lower the cerulein-induced pancreatitis. On the other hand, considering CAT and GST activities, EGCG was as effective as NAC in reducing oxidative stress.

Interaction between oxidative stress and cytokine release plays an important role in the systemic response of AP (34). In AP, it was known that pro-inflammatory cytokine levels, including TNF-a, IL-1 and IL- 6 increase, and these pro-inflammatory cytokines may play pivotal role in initiating the systemic response (35). The results of the present study showed that proinflammatory cytokine levels increased in response to pancreatitis induction. NAC treatment as an antioxidant agent caused decreases in serum IL-6 levels. Additionally, EGCG treatment seemed to be more effective in balancing IL-6 levels. However, serum TNF-a results showed a statistically significant increase in serum TNF-a level at a higher degree, compared to the results of the EGCG treatment group, relative to pancreatitis. It has been mentioned previously that when the AP is induced, pancreas inflammation begins in 3 hours and leukocyte recruitment, and pro-inflammatory cytokines are released by activated macrophages as a result of pancreatic damage (34). Increased levels of pro-inflammatory cytokines were reported to be correlated with the pancreatic inflammation stage (36). TNF- $a$ and IL-1 $\beta$ are two of the cytokines that initiate the systemic inflammatory response in AP, and they are known to amplify cascades of inflammatory response (37). In our cerulein-induced pancreatitis model, histopathological results were consistent with the model. However, while NAC treatment ameliorated the inflammatory response, the inflammatory response in the EGCG treated group seemed to be more severe than in the pancreatitis group. As well, in the inflammation sites of the pancreas, it was known that $\mathrm{T}$ cells take place in a smaller number in addition to macrophages. In the present study, increased number of CD8- $a$ positive cells, which is a marker of cytotoxic T cells, were observed in the cerulein-induced pancreatitis group, but EGCG treatment made the situation worsen by increased number of cytotoxic $T$ cells. Similar results could be seen for macrophages with staining of CD68+ cells. EGCG treatment caused increases in macrophage 
numbers. Recently, it was indicated that activated macrophages have an important role in decreasing the inflammation, and prompting wound healing, fibrosis as well as tumorigenesis. Fibrosis is reported to be regulated mainly by macrophages (38). Additionally, increases in the serum TNF-a level in EGCG groups were consistent with increased numbers of apoptotic cells. Accordingly, in several cells it was proved that TNF-a stimulate apoptotic/necrotic cell death (39). The results of previous studies conducted with cerulein-induced pancreatitis model, TNF-a mediated apoptosis was one of the main findings (39). Moreover, in the present study it may be suggested that EGCG treatment may be the aggravating factor, and may worsen pancreatitis for the cerulein-induced pancreatitis model.

EGCG, which is one of the most effective catechin compounds, mainly found in green tea leaves, has numerous health benefits, and these were attributed to having free radical scavenging properties, as well as being highly antioxidant. Green tea extract, which has numerous polyphenols, has been reported to ameliorate cerulein-induced male mice by inactivation of NF-$B$ inactivation and the oxidative stress pathway (11). However, in our study we aimed to evaluate especially EGCG, and our results were not consistent with the previous study. Although having beneficial effects, previous studies concluded that green tea extracts, such as EGCG, might have toxic and pro-oxidant effects (40). It was claimed that EGCG may induce apoptosis by the production of $\mathrm{H}_{2} \mathrm{O}_{2}$ in human cancer cell line $\mathrm{H661}$ (41). EGCG was reported to induce apoptosis in pancreatic cancer by caspase activation and mitochondrial membrane depolarization (42). Catechins are antioxidants that scavenge the free radicals, but also they were reported to induce oxidative stress, and such pro-oxidant effects were reported to induce apoptosis in cancer cells (19). One of previous studies declared that EGCG had strong ROS scavenging activity and protective effects at 2-30 mM concentrations; however, it was found that in concentrations higher than $60 \mathrm{mM}$, EGCG increased the oxidative damage on DNA. It was suggested that, EGCG had protective effects at lower concentrations; on the contrary, it was reported that at higher concentrations, EGCG exerted prooxidant effects on DNA by increased $\operatorname{ROS}(43,44)$. In summary, in the cancer therapy, green tea polyphenols may be a good alternative for consumption. Although, its protective effects were proved for cancer treatment strategies, the present study suggests that it is not an alternative treatment for AP.

\section{CONCLUSION}

In conclusion, although pro-oxidant effects of EGCG may induce apoptosis in tumorigenic cells and used for cancer therapy, when it comes to pancreatitis, EGCG may worsen the degree of pancreatitis. That may be attributed to the use of higher doses of EGCG. It can be concluded that overconsumption of polyphenols having pro-oxidant effects, including EGCG, may worsen pancreatitis conditions. Further analyses need to be performed in order to exert the pro-oxidant mechanisms and the dose dependency of action of EGCG for AP.
Peer-review: Externally peer-reviewed.

Author Contributions: Conception/Design of study: T.B.; Data Acquisition:T.B., G.G., E.K., G.S.; Data Analysis/Interpretation: E.K., G.G.; Drafting Manuscript: E.K., G.G.; Critical Revision of Manuscript: G.S., T.B.; Final Approval and Accountability: T.B., G.G., E.K., G.S.

Conflict of Interest: The authors declare that they have no conflicts of interest.

Financial Disclosure: This study was financialiy supported by the Hacettepe University Research Projects Coordination Unit (Project no: 09 D03 301 001).

\section{REFERENCES}

1. Lei QC, Wang XY, Xia XF, Zheng HZ, Bi JC, Tian F, et al. The role of omega-3 fatty acids in acute pancreatitis: A meta-analysis of randomized controlled trials. Nutrients 2015; 7(4): 2261-73.

2. Jha RK, Ma Q, Sha H, Palikhe M. Acute pancreatitis: a literature review. Med Sci Mon 2009; 15(7): RA147-RA56.

3. Aranda-Narváez JM, González-Sánchez AJ, Montiel-Casado MC, Titos-García A, Santoyo-Santoyo J. Acute necrotizing pancreatitis: surgical indications and technical procedures. World J Clin Cases 2014; 2(12): 840.

4. Elfar M, Gaber LW, Sabek O, Fischer CP, Gaber AO. The inflammatory cascade in acute pancreatitis: relevance to clinical disease. Surg Clin North Am 2007; 87(6): 1325-40.

5. Saruç M, Yuceyar H, Turkel N, Ozutemiz O, Tuzcuoglu I, Ayhan S, et al. The role of heme in hemolysis-induced acute pancreatitis. Med Sci Mon 2007; 13(3): BR67-BR72.

6. Wei M, Gong Y-J, Tu L, Li J, Liang Y-H, Zhang Y-H. Expression of phosphatidylinositol-3 kinase and effects of inhibitor wortmannin on expression of tumor necrosis factor-a in severe acute pancreatitis associated with acute lung injury. World J Emerg Med 2015; 6(4): 299.

7. De Campos T, Deree J, Coimbra R. From acute pancreatitis to endorgan injury: mechanisms of acute lung injury. Surgical Infect 2007; 8(1): 107-20.

8. Mokra D, Kosutova P. Biomarkers in acute lung injury. Respir Physiol Neurobiol 2015; 209: 52-8.

9. Tsuji N, Watanabe N, Okamoto T, Niitsu Y. Specific interaction of pancreatic elastase and leucocytes to produce oxygen radicals and its implication in pancreatitis. Gut 1994; 35(11): 1659-64.

10. $\mathrm{Yu} \mathrm{JH}, \mathrm{Kim} \mathrm{H}$. Oxidative stress and inflammatory signaling in cerulein pancreatitis. World journal of gastroenterology: WJG 2014; 20(46): 17324.

11. Babu BI, Malleo G, Genovese T, Mazzon E, Di Paola R, Crisafulli C, et al. Green tea polyphenols ameliorate pancreatic injury in ceruleininduced murine acute pancreatitis. Pancreas 2009; 38(8): 954-67.

12. Su KH, Cuthbertson C, Christophi C. Review of experimental animal models of acute pancreatitis. HPB (Oxford). 2006; 8(4): 264-86.

13. Pandey KB, Rizvi SI. Plant polyphenols as dietary antioxidants in human health and disease. Oxid Med Cell Longev 2009; 2(5): 270-8.

14. Shay J, Elbaz HA, Lee I, Zielske SP, Malek MH, Hüttemann M. Molecular mechanisms and therapeutic effects of (-)-epicatechin and other polyphenols in cancer, Inflammation, Diabetes, and Neurodegeneration. Oxid Med Cell Longev 2015; 2015.

15. Erguder IB, Avci A, Devrim E, Durak I. Effects of cooking techniques on antioxidant enzyme activities of some fruits and vegetables. Turk J Med Sci 2007; 37(3): 151-6. 
16. Mekiňová D, Chorvathova V, Volkovova K, Staruchova M, Graňičová E, Klvanova J, et al. Effect of intake of exogenous vitamins C, E and $\mathrm{B}$-carotene on the antioxidative status in kidneys of rats with streptozotocin-induced diabetes. Mol Nutr Food Res 1995; 39(4): 257-61.

17. Süloğlu AK, Girgin G, Selmanoğlu G, Balcı S, BaydarT. Possible effects of lycopene and silymarin on rat liver functions and oxidative stress markers. Turk J Biochem/Turk Biyokim Derg 2014; 39(3):344-50.

18. Sureda A, Tejada S, Del Mar Bibiloni M, Antoni Tur J, Pons A. Polyphenols: well beyond the antioxidant capacity: polyphenol supplementation and exercise-induced oxidative stress and inflammation. Curr Pharm Biotechnol 2014; 15(4): 373-9.

19. Lambert JD, Elias RJ. The antioxidant and pro-oxidant activities of green tea polyphenols: a role in cancer prevention. Arch Biochem Biophys 2010; 501(1): 65-72.

20. Lin C-H, Chao L-K, Hung P-H, Chen Y-J. EGCG inhibits the growth and tumorigenicity of nasopharyngeal tumor-initiating cells through attenuation of stat3 activation. Int J Clin Exp Pathol 2014; 7(5): 2372.

21. Yu H, Pardoll D, Jove R. Stats in cancer inflammation and immunity: a leading role for stat3. Nat Rev Cancer 2009; 9(11): 798-809.

22. Mukherjee S, Siddiqui MA, Dayal S, Ayoub YZ, Malathi K. Epigallocatechin-3-gallate suppresses proinflammatory cytokines and chemokines induced by toll-like receptor 9 agonists in prostate cancer cells. J Inflamm Res 2014; 7: 89.

23. Ramudo L, Manso MA. N-acetylcysteine in acute pancreatitis. World J Gastrointest Pharmacol Ther 2010; 1(1): 21.

24. Muià C, Mazzon E, Di Paola R, Genovese T, Menegazzi M, Caputi AP, et al. Green tea polyphenol extract attenuates ischemia/reperfusion injury of the gut. Naunyn-Schmiedebergs Arch Pharmacol 2005; 371(5): 364-74.

25. Senthil Kumaran V, Arulmathi K, Srividhya R, Kalaiselvi P. Repletion of antioxidant status by EGCG and retardation of oxidative damage induced macromolecular anomalies in aged rats. Exp Gerontol 2008; 43(3): 176-83.

26. Meng M, Li Y-Q, Yan M-X, Kou Y, Ren H-B. Effects of epigallocatechin gallate on diethyldithiocarbamate-induced pancreatic fibrosis in rats. Biol Pharm Bull 2007; 30 (6): 1091-6.

27. Abdallah MF, Karacaoglu E, Girgin G, Kilicarslan B, Selmanoglu G, Baydar T. Influence of subacute melatonin treatment on antioxidant factors in the liver of female rats. J Exp App Anim Sci 2015; 1(3): 359-68.

28. Yagci G, Gul H, Simsek A, Buyukdogan V, Onguru O, Zeybek N, et al. Beneficial effects of $\mathrm{N}$-acetylcysteine on sodium taurocholateinduced pancreatitis in rats. J Gastroenterol 2004; 39(3): 268-76.

29. Kim H. Cerulein pancreatitis: oxidative stress, inflammation, and apoptosis. Gut Liver 2008; 2(2): 74-80.

30. Hyun JJ, Lee HS. Experimental models of pancreatitis. Clin Endosc 2014; 47(3): 212-6.
31. Rodrigo R, Gil-Becerra D. Chapter 17 - Implications of polyphenols on endogenous antioxidant defense systems in human diseases. Polyphenols in Human Health and Disease. San Diego: Academic Press; 2014. p. 201-17.

32. Fukui M, Kanoh M, Takamatsu Y, Arakawa Y. Analysis of serum catalase activities in pancreatic diseases. J Gastroenterol 2004; 39(5): 469-74.

33. Rahman SH, Ibrahim K, Larvin M, Kingsnorth A, Mcmahon MJ. Association of antioxidant enzyme gene polymorphisms and glutathione status with severe acute pancreatitis. Gastroenterology 2004; 126(5): 1312-22.

34. Pérez S, Pereda J, Sabater L, Sastre J. Redox Signaling in acute pancreatitis. Redox Biol 2015; 5: 1-14.

35. Gomez-Cambronero LG, Sabater L, Pereda J, Cassinello N, Camps B, Vina J, et al. Role of cytokines and oxidative stress in the pathophysiology of acute pancreatitis: therapeutical implications. Curr Drug Targets Inflamm Allergy 2002; 1(4): 393-403.

36. Mayer J, Rau B, Gansauge F, Beger HG. Inflammatory mediators in human acute pancreatitis: clinical and pathophysiological implications. Gut 2000; 47(4): 546-52.

37. Javier E, Javier P, Alessandro A, Juan S, Luis S, Luis A, et al. Role of redox Signaling, protein phosphatases and histone acetylation in the inflammatory cascade in acute pancreatitis: therapeutic implications. Inflamm Allergy Drug Targets 2010; 9(2): 97-108.

38. Xue J, Sharma V, Hsieh MH, Chawla A, Murali R, Pandol SJ, et al. Alternatively activated macrophages promote pancreatic fibrosis in chronic pancreatitis. Nat Commun 2015; 6: 7158.

39. Gukovskaya AS, Gukovsky I, Zaninovic V, Song M, Sandoval D, Gukovsky S, et al. Pancreatic acinar cells produce, release, and respond to tumor necrosis factor-alpha. Role in regulating cell death and pancreatitis. J Clin Invest 1997; 100(7): 1853-62.

40. Elbling L, Weiss R-M, Teufelhofer O, Uhl M, Knasmueller S, SchulteHermann R, et al. Green tea extract and (-)-epigallocatechin-3-gallate, the major tea catechin, exert oxidant but lack antioxidant activities. The FASEB J 2005; 19(7): 807-09.

41. Yang G-Y, Liao J, Li C, Chung J, Yurkow EJ, Ho C-T, et al. Effect of black and green tea polyphenols on C-Jun phosphorylation and $\mathrm{H}_{2} \mathrm{O}_{2}$ production in transformed and non-transformed human bronchial cell lines: possible mechanisms of cell growth inhibition and apoptosis induction. Carcinogenesis 2000; 21(11): 2035-9.

42. Qanungo S, Das M, Haldar S, Basu A. Epigallocatechin-3-gallate induces mitochondrial membrane depolarization and caspasedependent apoptosis in pancreatic cancer cells. Carcinogenesis 2005; 26(5): 958-67.

43. Hayakawa F, Ishizu Y, Hoshino N, Yamaji A, Ando T, Kimura T. Prooxidative activities of tea catechins in the presence of $\mathrm{Cu}^{2+}$. Biosci Biotechnol Biochem 2004; 68(9): 1825-30.

44. Preedy VR. Tea in health and disease prevention: Academic Press; 2012. 